\title{
Development and Prospect of 4g and 5g Technology
}

\author{
Li-qiang Ni \\ ${ }^{I}$ (School of Electronic and Information Engineering, Tianjin Poly-technic University, China)
}

\begin{abstract}
With the development of science and technology, the mobile communication technology has a huge change. Such as, the mobile technology of $3 G$, which was used widely by people, will disappear slowly in the near future. However, the $4 G$ and $5 G$ will be study and then will be widely used. The fifth generation mobile communication system $(5 G)$ is a new generation of mobile communication system after 2020 and its technological development is still in the exploratory stage. In this paper, we will talk about the key elements of this hot technology-4G and $5 G$.
\end{abstract}

Keywords: $4 G, 5 G$, mobile communication technology, mobile phone, mobile communication tools

\section{Introduction}

Cell phone is the most comment machine in our daily life, which has been playing a more and more important part. Thus, the progress of mobile communication technology has a close relationship with us. Nowadays, with the development of science and technology, the mobile communication technology has a huge innovation. From $2 \mathrm{G}$ to $3 \mathrm{G}$, and $4 \mathrm{G}, 5 \mathrm{G}$, mobile communication technology has been innovated continuously ${ }^{[1]}$. In December 2013, 4G was allowed to enter the market for public in China. However, at the same time, the research of 5G, the new generation representative of mobile communication system which has a faster update speed, has been devoted in many developed countries. The technology of $4 \mathrm{G}$ and $5 \mathrm{G}$ comes into our vision one by one.

Comparing data transfer rates from mobile communication systems as shown in Figure 1:

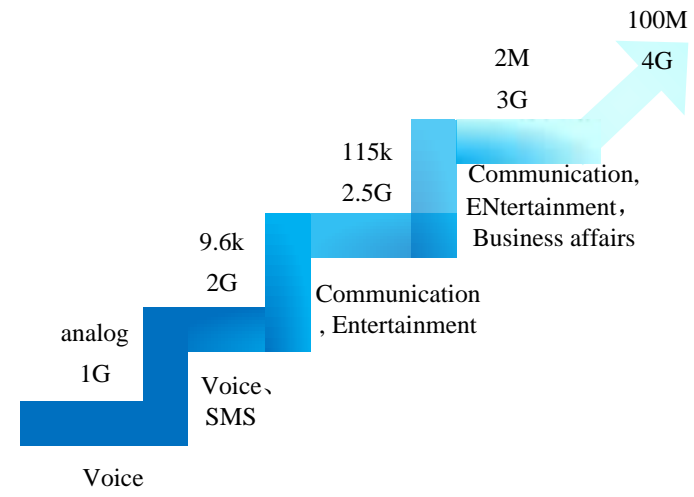

Figure 1 Comparing data transfer rates from mobile communication systems

1. The first generation of analog only provides voice services.

2. The second generation digital mobile communication system transmission rate is only $9.6 \mathrm{Kbps}$, up to $32 \mathrm{Kbps}$, such as PHS.

3. Third generation mobile communication system data transmission rate can reach $2 \mathrm{Mbps}$.

4. Fourth generation mobile communication system data transmission rate can reach $20 \mathrm{Mbps}$, or even up to $100 \mathrm{Mbps}$.

So what should we know is, how many technology does the 4G and 5G have?

\section{Technology of $\mathbf{4 G}$}

The 4th generation mobile communication technology ${ }^{[2]}$, $4 \mathrm{G}$ short for, comparing with $3 \mathrm{G}$, it has many advantages, such as: the faster speed of transfer, the higher transfer quality and the lower cost. The technologies of 4G include multiple access techniques, Smart antenna technology, software defined radio (SDR) and etc...

The following detailed description of the key features of $4 \mathrm{G}$ Technology:

$4 \mathrm{G}$ adopts the orthogonal frequency division multiplexing $(\mathrm{OFDM})^{[3]}$, Schematic diagram of OFDM as figure 2, from multiple access techniques; the mean idea is dividing the information channels in order to improve the utilization of the channel. Some details as follow, dividing the original channel, and then let the latter transform respectively, parallel arrival. In this way, the road of signal is flat, but the bandwidth (BW) is 
small. So it can strengthen the ability of anti-jamming, however, it also brings some influence towards the system complexity.

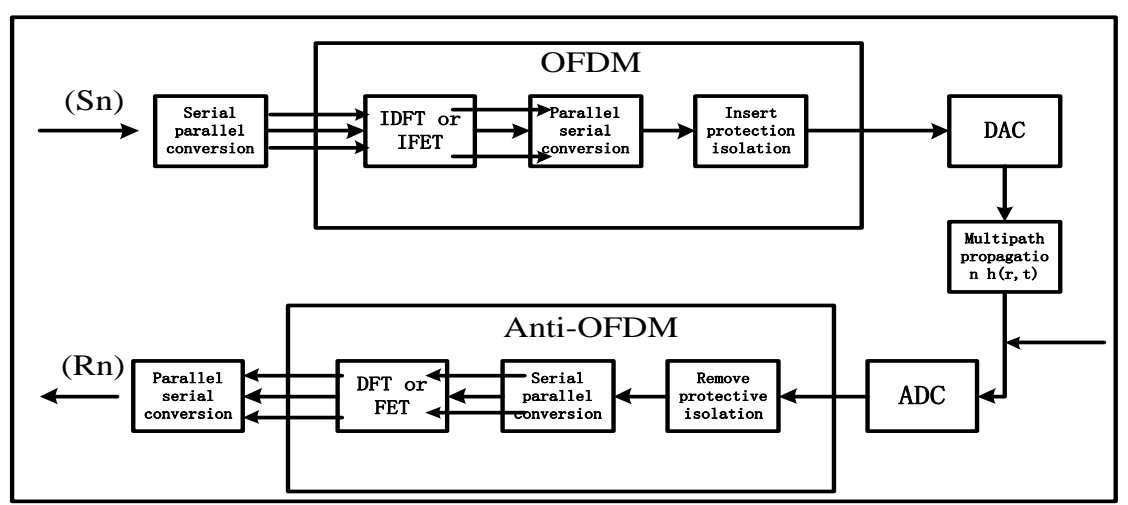

Figure 2 Schematic diagram of OFDM

Smart antenna technology (show as figure 3) is a multi-beam or adaptive array antenna, which doesn't switch between beams; it can achieve the suppressing signal interference, automatic tracking and adjustable digital beam. According to the signal wave and emission direction, it can adjust by itself, and can distinguish the relative synchronization signal dynamically. Adjust the signal to the region dynamically, and the direction of beam you want to arrive is constant, what's more, the beam fringe interference doesn't affect the main beam. Appling digital signal processing technology to produce spatial directional beam, signal interference is a problem that every generation of communication system want to solve. In addition, the research and development are not separated from reducing noise by every technology. Nowadays, Smart antenna is a better way to reduce noise compare with others. On the one hand, it can improve the capacity of the system largely, ensure the quality of signal. On the other hand, expanding the coverage area and having a lower cost.

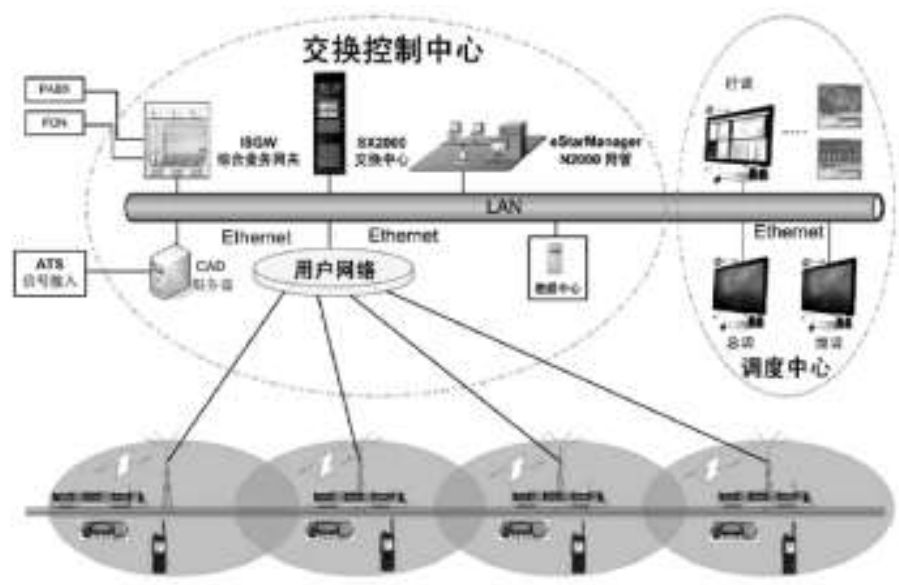

Figure 3 Frame of smart antenna technology

Software defined radio (SDR) is a wireless communication mode, using software to achieve physical layer link, adopting digital signal processing technology, and completing it's corresponding function by software instead of hardware. The main principle is using the broadband wireless receiver to replace the narrow one, and put the DAC around the antenna, completing setting of signal rule by software. The structure of SDR is universal, with some advantages, such as: achieving functions flexibly, updating conveniently, and enhancing the ability of anti-jamming from system. In addition, it can be suit the different Network and interface.

There is not compatibility between the system of $4 \mathrm{G}$ and others. It can complete the access of signal and all tasks of IP by point to point independently, and can communicate each other with the core network. This open structure has brought many conveniences: the converting interface for technology and equipment can be cancel. Independent IP technology enables wireless access methods and physical layers to be independent of each other that interdependent originally. IP core network is compatible with a variety of wireless access protocols, which don't need to be considered when designing the core network, and it is very flexible. In addition, there is a high performance for $4 \mathrm{G}$ system.

III. Difference of 4G and $5 G$ 
At present, 4G has already entered the intensive stage of popularization and application, most mobile phones start to support 4G networks, such as Huawei, iPhone and Samsung, and we can experience the increase of $4 \mathrm{G}$ network speed. However, with the development of technology and the expansion of the future demand, $5 \mathrm{G}$ has entered research phase in full swing, the era of $4 \mathrm{G}$ gradually shifted to 5G Market and 5G technology is newer and faster than $4 \mathrm{G}$.

In the future, $5 \mathrm{G}$ will be able to bring us faster mobile experience of the Internet and more mobile applications, for example, domestic appliances and even cars will be controlled by remote manipulation of mobile phone, realizing the interconnection of all things. In communication industry, 5G also means that the technical standards and patent rights to compete in the future. Each generation of communication network will form a number of intellectual property rights represented by patents.

The number of manufacturers involved will affect how much of their profits from the industry in the coming years. Huawei's intellectual property rights have in the $4 \mathrm{G}$ technology reached $25 \%, 5 \mathrm{G}$ is expected to achieve more patents. The application and popularization of the new communication technology generation will also bring a new round of investment boom.

From the perspective of technology, $5 \mathrm{G}$ is not a revolution, and $5 \mathrm{G}$ is a continuation of $4 \mathrm{G}, 5 \mathrm{G}$ network will not have much modification in the core network, its key technologies are concentrated in the wireless part. There is no final conclusion on what technology will be used, and there is a brief introduction about some of the techniques currently used.

\section{4.5G lead the scale of the Internet of things business; 5G open the door to the Internet of all things}

4.5G is the evolution of $4 \mathrm{G}$, can provide XGbps large capacity, 10ms low latency and $>300$ billion connections based on SOMA, 256QAM, Massive MIMO and other key technologies to provide XGbps high capacity. $4.5 \mathrm{G}$ is a full range of smooth evolution of $4 \mathrm{G}$, can be achieved on the existing $4 \mathrm{G}$ software upgrades or add some hardware to achieve, $4.5 \mathrm{G}$ positioning in the next five years, the emergence of new terminals, new business, new experience, is the forerunner of $5 \mathrm{G}$. Figure 4 is the difference of $4 \mathrm{G}, 4.5 \mathrm{G}$ and $5 \mathrm{G}$.

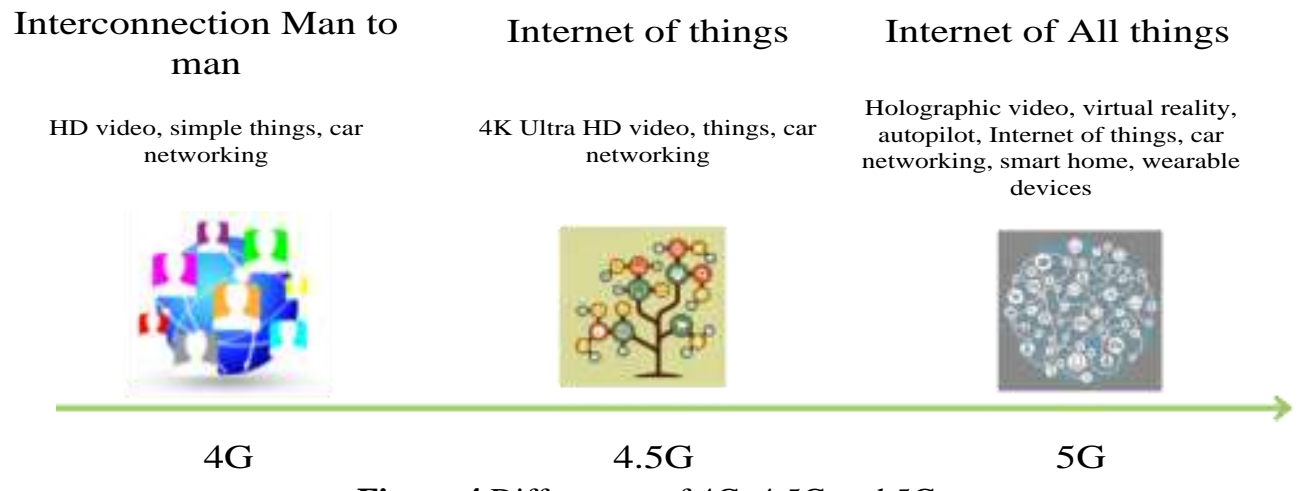

Figure 4 Differences of $4 \mathrm{G}, 4.5 \mathrm{G}$ and $5 \mathrm{G}$

5G is not only a technical upgrade; it will build a broad platform for us, the birth of numerous new applications, new industries. 5G will be an important infrastructure and key enabler for all connected world and future information society. The current $4.5 \mathrm{G}$ standard R12 will be frozen at the end of this year, R13 standards are being developed; $5 \mathrm{G}$ standards are in the research phase. Figure 5 shows $4.5 \mathrm{G}, 5 \mathrm{G}$ standard progress

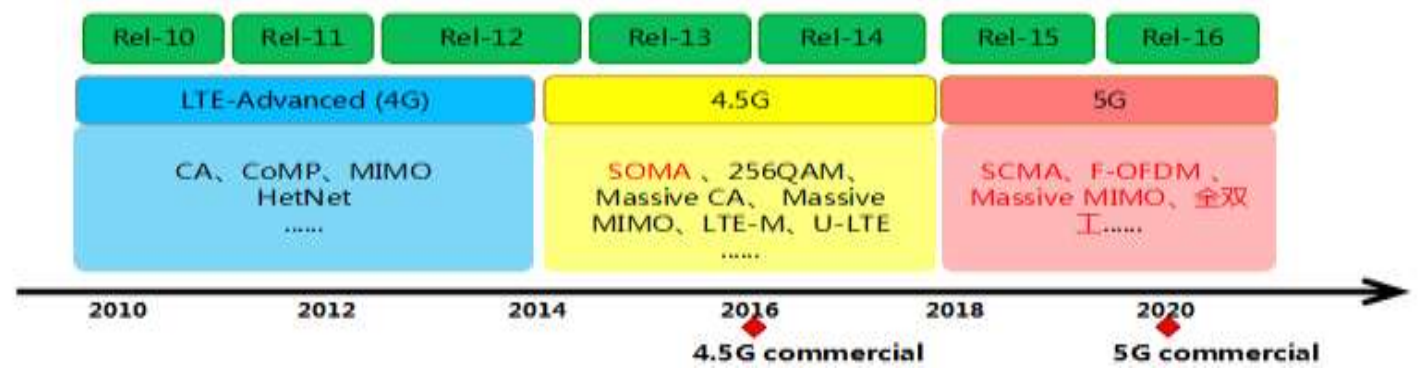

Figure 5 4.5G, 5G standard progress: expected 4.5G commercial in 2016, commercial 5G in 2020

\section{Technology of 5G}


$5 \mathrm{G}$ turns to vast space of high frequency instead of low frequency field, making spectrum resources rich, such as $\mathrm{cm}$ band and millimeter wave band resources can be used. The field of spectrum develops the design and function of technology, and improves the speed of the short distance communication ${ }^{[4]}$.

SCMA is a new multiple access technology, in the time domain, frequency domain, based on the increase in the domain of code reuse, improve spectrum efficiency and system capacity. SCMA compared to OFDMA can enhance $300 \%$ gain, as shown in figure 6 . And a table (table 1) has been shown to compare with SCMA and OFDMA.

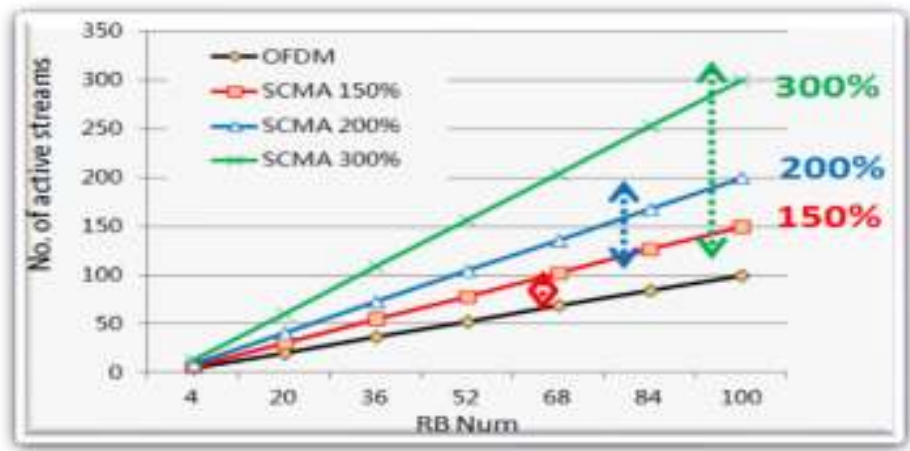

Figure 6 SCMA compared to OFDMA can enhance 300\% gain

Table 1 SCMA compared to OFDMA

\begin{tabular}{|l|l|}
\hline Mode & SCMA over OFDM \\
\hline UE & Max 12 active UE, 1 Tx/UE \\
\hline eNB & $1 \mathrm{Tx}, 2 \mathrm{Rx}$ \\
\hline System BW & $20 \mathrm{MHz}$ \\
\hline RB per UE & (48 RBs for 12 SCMA Users with 300\% overloading) \\
\hline Link & TDD-UL, PUSCH \\
\hline
\end{tabular}

F-OFDM is a new waveform, empty by F-OFDM, can realize the different attributes of the business based on the flexible allocation of time frequency resource, is the basis for realization of adaptive air interface. F-OFDM supports different waveforms, multiple access technology, TTI access, as figure 7 showed.

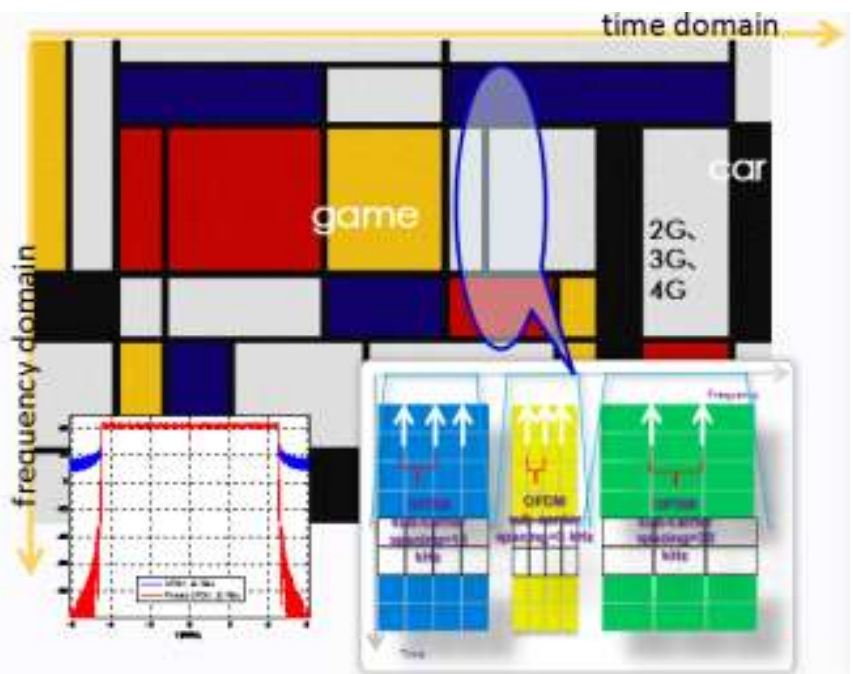

Figure 7 F-OFDM supports different waveforms, multiple access technology, TTI access

The new technology of antenna is the technology of 5G exploration. The new technology of multiple antennas has been researched in the previous, passive and active technologies have rich development and practice experience, including 2D technology and 3D technical supports. With the development of science and technology, the efficiency of spectrum will be improved, and we are looking forward to get greater breakthroughs of rate. The previous generations of communication technology has been used in network mode, and it is a cellular communication system, the base station covers the center area. And the base station is unable to move, the structure is not flexible, $5 \mathrm{G}$ may never use a similar base station. 
$5 \mathrm{G}$ is exploring a full-duplex technology. On the same signal, sending and receiving signals at the same time, the efficiency will be double. 5G technology will become more diverse, more comprehensive, more intelligent, adapting for the growth of data in the future. As one of the main using of technology in the futureRAN access network architecture, it can access the central treatment and real-time cloud computing function. The main research contents include the architecture and function of C-RAN, such as centralized control, baseband pool RRU interface definition, based on the C-RAN closer collaboration. Similarly, the base station clusters and virtual community is research and development.

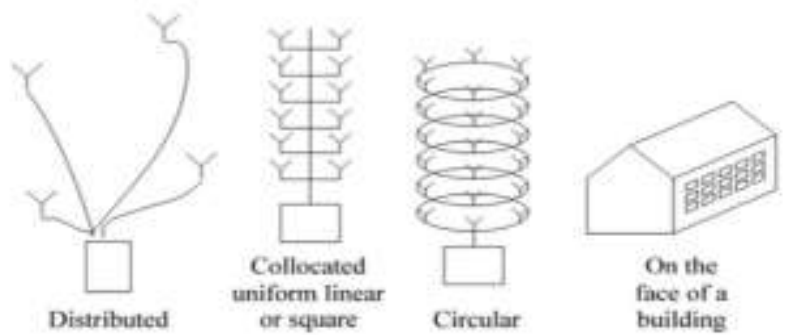

Figure 8 some of the possible configurations and deployment scenarios for the Massive MIMO base station

The figure 8 above shows some of the possible configurations and deployment scenarios for the Massive MIMO base station. Massive MIMO can increase the capacity by 10 times or more because of its active spatial multiplexing. At the same time, through a large number of antennas, the energy can be gathered in the small area of the space, thus improving the energy efficiency. That is its characteristic.

In recent decades, communication tools are changing our life and making our work and life more and more close, such as sending a message, calling somebody, playing games, watching video and so on, more and more functions will be used in mobile phones, mobile phone will become the center of all the equipment command .Mobile phone brings great convenience to our life, many industry made the client for the mobile phone users, browsing through the network, online shopping, online learning, bank transactions, mobile phones navigation and queries, etc. Mobile phone app is very convenient for the client, favored by users, the demand of the mobile phone function in the future will be more and more large, so phone carries on so many functions, the speed of the communication network support, quality support is also greatly tests the research and development personnel of $5 \mathrm{G}$, deciding to bring us what kind of $5 \mathrm{G}$ in the future. Therefore, all the technology supports rate, noise reduction and security without exception. Using the D2D to realize direct communication, research and development of new network architecture is $5 \mathrm{G}$.

\section{Conclusion}

Although there is not the established standard of 5G, when the 4G are used in China, the technology of 5G has been being studying in developed countries, and a lot of money has been devote to this item. In fact, $5 \mathrm{G}$ is not only a technology to display the science and the new technology, but the amalgamation of $3 \mathrm{G}$ and $4 \mathrm{G}$; it is a large amalgamation of the mobile communication technology. It's a trend to develop mobile communication technology, to develop our life. Finally, let's guess the tomorrow of $5 \mathrm{G}$ and our life. What the achievement we can get and the development of communication technology in China? Let us look forward to.

\section{Acknowledgements}

This paper is supported by the School of Electronic and Information Engineering of the Tianjin Polytechnic University.

\section{References}

[1]. Zhu-hu Xiao, Development trend and key technologies of 5G mobile communication, Chinese Science, 2014, (5).

[2]. Xian-ying Zhang, Technical analysis of the 4th generation mobile communication technology, Digital Communication World, 2011, (6)

[3]. WU Wei-chi, ZHANG Duo-ying. Research on the key technologies of optical orthogonal frequency division multiplexing [J].Optical Communication Technology, 2013, (11):5-8

[4]. Boccardi F, Heath R W, Lozano A, et al. Five disruptive technology directions for 5G[J]. Communications Magazine IEEE, 2014, 52(2):74-80 